BASFI, BASMI and ASDAS were found to be similar in both groups. Median BASDAI scores at first TNFi initiation were higher in patients with nr-axSpA than in patients with AS $(58.65 \pm 18.21,51.06 \pm 18.91, p=0.030)$. Cumulative drug survival rates did not show significant difference among INF (at 59. months:18,5\%), ADA (at 71. months: $39,5 \%$ ) and ETN (at 51. months: $24,2 \%$ ) in nr-axSpA group $(p=0,699$ ) (figure 1). Similarly, drug survival rates at $78,77,78$. months for 3 antiTNF drugs had shown no difference in AS patients (INF (at 78. months: $38,1 \%$ ), ADA (at 77. months: $52,4 \%)$, ETN (at 78. months: $39,0 \%)$ ) $(p=0,151)$ (Figure 2). Cumulative survival rates in AS patients (at 78. months:42,2\%) were found to be significantly higher than that (at 71 . months:28,2\%) in $n r-a x S p A$ patients $(p<0,001)$ (Figure 3).
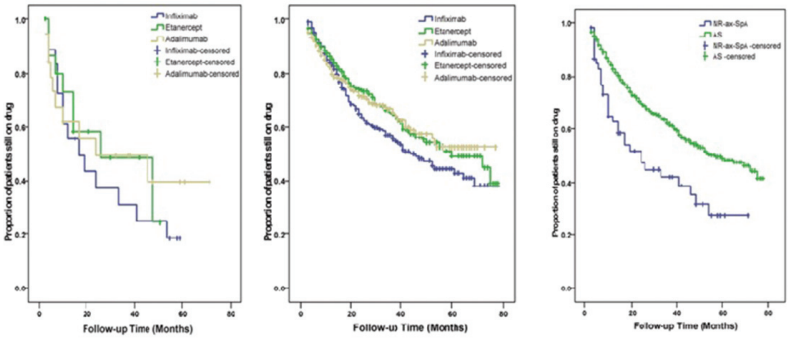

Abstract AB0835 - Figure 1. Drug survival rates anti-TNF in nr-axSpA. Abstract AB0835 Figure 2. Drug survival rate by anti-TNF in AS. Abstract AB0835 - Figure 3. Overall drug survival on first anti-TNF in nr-axSpA and AS patients.

Conclusions: In contrast to the literature that revealed similar short term survival rates for anti-TNF drugs in patients with AS and nr-axSPA, we found higher survival rates in patients with AS compared to patients with nr-axSpa in this long-term observational study.A limitation of the study may be the low number of nr-axSpa patients using anti-TNF, related to the requirements of social insurance system. Disclosure of Interest: None declared

DOI: 10.1136/annrheumdis-2018-eular.7023

\section{AB0836 DRUG RETENTION RATE OF THE FIRST TNF INHIBITOR IN RADIOGRAPHIC AND NON RADIOGRAPHIC AXIAL SPONDYLOARTHRITIS: DATA FROM A MULTICENTER STUDY}

G. Lopalco ${ }^{1}$, V. Venerito ${ }^{1}$, L. Cantarini ${ }^{2}$, G. Emmi $^{3}$, F. Salaffi ${ }^{4}$, S. Tafuri ${ }^{1}$, F. P. Bianchi ${ }^{1}$, M. Di Carlo ${ }^{4}$, S. Gentileschi ${ }^{2}$, G. Di Scala ${ }^{3}$, M. Nivuori' ${ }^{1}$, S. Perniola ${ }^{1}$, M. Fornaro ${ }^{1}$, M.G. Anelli ${ }^{1}$, F. Cacciapaglia ${ }^{1}$, C. Scioscia ${ }^{1}$, C. Fabiani ${ }^{5}$ M. Galeazzi ${ }^{2}$, G. Lapadula ${ }^{1}$, F. lannone ${ }^{1}$. 'University of Bari, Bari; ${ }^{2}$ University of Siena, Siena: ${ }^{3}$ University of Florence, Firenze: ${ }^{4}$ Università Politecnica delle Marche, Jesi; ${ }^{5}$ Humanitas Clinical and Research Center, Rozzano, Italy

Background: Good survival rates of TNF inhibitors (TNFi) have been shown in patients affected with axial Spondyloarthritis (axSpA) treated in a real-life setting, irrespective of administered anti-TNF agent. ${ }^{1}$ Although the use of these drugs in patients with non radiographic axSpA (nr-axSpA) remains topic of debate, ${ }^{2}$ several RCTs support their employment in these patients. ${ }^{3}$

Objectives: To assess the retention rate of the first TNF inhibitor between axSpA and $\mathrm{nr}-\mathrm{axSpA}$ patients. Additional aims: i) to evaluate any difference in persistence rates between anti-TNF monoclonal antibodies and etanercept (ETN) ii) to assess any impact of clinical, therapeutic and demographic features as well as radiographic findings on drug survival.

Methods: We retrospectively assessed 221 patients with axSpA, all fulfilling the ASAS criteria, who underwent first line therapy with TNFi from January 1st 2012 to September 30th 2016. Clinical, therapeutic and demographic features as well as radiographic findings were recorded at baseline and at the moment of therapy discontinuation. 126/221 patients (57.01\%) were treated with Adalimumab, 45/ 221 with ETN, 22/221 with Infliximab, 20/221 (9.05\%) with Golimumab, whereas $8 / 221(3.62 \%)$ with Certolizumab Pegol. Drug retention rates were analysed using Kaplan-Meier curves; log-rank test was performed to demonstrate differences in the survival function. Cox regression models were used to estimate the inference of several clinical and disease characteristics on drug discontinuation. Goodness of fit of the final model was assessed comparing Cox-Snell residuals to NelsonAlen cumulative hazard function.

Results: Drug survival in first line therapy was significantly lower in patients who had $n r-a x S p A$ than in those with radiographic sacroilitis $(p=0.005$, figure 1$)$ whereas survival rate did not differ significantly between patients treated with ETN and anti-TNF $\alpha$ monoclonal antibodies $(\mathrm{p}=0.057)$. Multivariate Cox model shown that nr-axSpA (HR 1.90), higher BMI (HR 1.18), higher HAQ, (HR 1.82) and higher
BASDAI (HR 1.25), were predictors of drug discontinuation, Nelson-Alen hazard function following very closely Cox-Snell residuals for drug persistence in first line therapy showed that the final model fitted well the data, except for large values of time (figure 2).

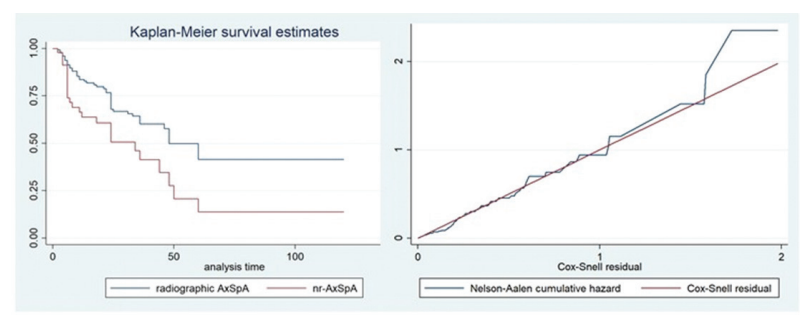

Abstract AB0836 - Figure 1. Kaplan-Meier curves. Abstract AB0836 - Figure 2. Cox-Snell residuals vs Nelson-Aalen cumulative Hazard function

Conclusions: Effectiveness of TNFi, estimated by drug survival, seems to be lower in patients with $\mathrm{nr}-\mathrm{AxSpA}$ than those affected with axSpA. The reason of these findings remain to be elucidated. However, a possible explanation may be searched in the limit of the classification criteria for $\mathrm{nr}$-axSpA. In addition overweight and high disease activity negatively impact the persistence on first line anti-TNF $\alpha$ treatment in axSpA patients in real life setting.

\section{REFERENCES}

[1] Favalli EG, et al. Arthritis Care Res (Hoboken). 2017 Jun;69(6):867-874.

[2] Vidal C, et al. Joint Bone Spine. doi:10.1016/j.jbspin.2017.08.005

[3]

F Sieper J et al. Ann Rheum Dis 72(6):815-822. doi:10.1136/annrheumdis-2012-201766

Disclosure of Interest: None declared

DOI: 10.1136/annrheumdis-2018-eular.3069

\section{AB0837 THE EFFECT OF ANTI-TNF ON RENAL FUNCTION IN PATIENTS WITH ANKYLOSING SPONDYLITIS: A PROSPECTIVE COHORT STUDY}

I.A. Swart, M. Heslinga, I.M. Visman, I.E. van der Horst-Bruinsma, C. van Denderen, M.T. Nurmohamed. AMSTERDAM RHEUMATOLOGY AND IMMUNOLOGY CENTER / READE, Amsterdam, Netherlands

Background: Impaired renal function is common in patients with ankylosing spondylitis (AS) and patients also have an increased risk of cardiovascular disease (CVD). Previous studies showed that biologicals, such as anti-Tumour Necrosis Factor (anti-TNF) reduce CVD in patients with inflammatory rheumatic disease. Impaired renal function is a known predictor of CVD (also elevated in AS). We postulated that the favourable cardiovascular effect of anti-TNF might be mediated by improving renal function. However, data about the effect of biologicals on renal function in patients with AS are lacking.

Objectives: To assess the effect of anti-TNF on renal function in patients with AS

Methods: Biological-naïve consecutive AS patients treated with etanercept or adalimumab were prospectively followed from 2005 to 2014. Renal function was determined by calculation of the estimated Glomerular Filtration Rate (eGFR) which was estimated with the abbreviated Modification of Diet in Renal Disease (MDRD) formula. Patients were divided into two groups: patients with normal rena function at baseline and patients with impaired renal function at baseline, to inves tigate whether the effect is different for these groups. Normal renal function was defined by eGFR $>90 \mathrm{~mL} / \mathrm{min} / 1.73 \mathrm{~m}^{2}$ at baseline and impaired renal function was defined by eGFR $<90 \mathrm{~mL} / \mathrm{min} / 1.73 \mathrm{~m}^{2}$ at baseline. The effect of anti-TNF on eGFR was analysed using mixed model analysis.

Results: 211 AS patients were followed for a median of $156(36-286)$ weeks 153 patients had normal renal function and 58 had impaired renal function a baseline. In patients with normal renal function at baseline eGFR decreased significantly over time $(\beta=-0.041, p=0.001)$, although this association did not remain significant after adjustment for disease activity $(\beta=-0.015, p=0.212)$. Patients with impaired renal function at baseline did not have a significant change in eGFR over time $(\beta=0.022, p=0.087)$ and this association remained not significant after adjustment for alcohol consumption, BMI, disease duration and disease activity $(\beta=0.008, p=0.593)$. The change in eGFR on average over time after starting antiTNF in AS patients with normal and impaired kidney function are presented in figure 1. 


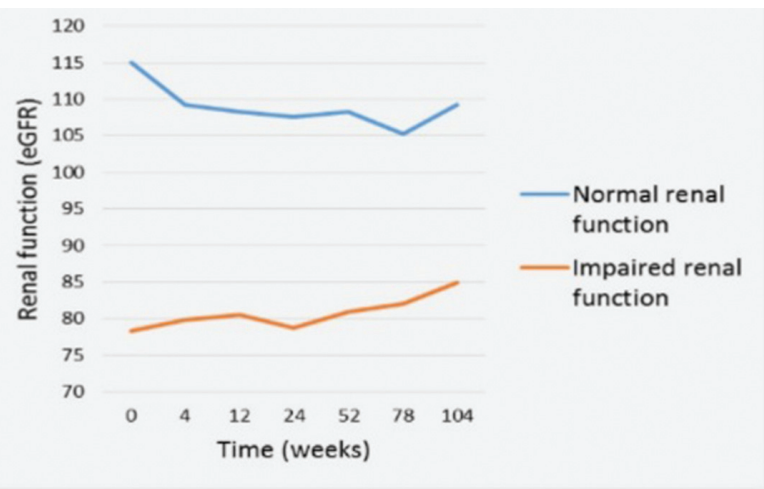

Abstract AB0837 - Figure 1

Conclusions: This study demonstrates that anti-TNF is not associated with renal function in AS patients, which means that use of anti-TNF is safe concerning renal function in patients with AS. From our results it seems that the effect of anti-TNF on CVD in AS patients is not mediated by an effect on renal function.

Disclosure of Interest: None declared

DOI: 10.1136/annrheumdis-2018-eular.6300

\section{AB0838 THE EFFICACY OF ADALIMUMAB AND SULFASALAZINE IN ALLEVIATING AXIAL AND AORTIC INFLAMMATION DETECTED IN PET/CT IN PATIENTS WITH AXIAL SPONDYLOARTHRITIS}

J.-P. Kaijasilta ${ }^{1}$, A. Kerola ${ }^{1}$, R. Tuompo ${ }^{2}$, M. Kauppi ${ }^{3}$, H. Relas ${ }^{2}$, A. Loimaala ${ }^{4}$, H. Koivu ${ }^{5}$, J. Schildt ${ }^{4}$, T. Kerola ${ }^{1}$, K. Eklund ${ }^{2}$, T. Nieminen ${ }^{6} .{ }^{1}$ Internal Medicine, Päijät-Häme Central Hospital, Lahti, ${ }^{2}$ Inflammation Center, Helsinki University Hospital, Helsinki; ${ }^{3}$ Rheumatology, University of Tampere School of Medicine, Tampere; ${ }^{4}$ Medical Imaging Center, Helsinki University Hospital, Helsinki; ${ }^{5}$ Department of Nuclear Medicine, Päijät-Häme Central Hospital, Lahti; ${ }^{6} \mathrm{Heart}$ and Lung Center, Helsinki University Hospital, Helsinki, Finland

Background: Inflammatory pathways are likely the central link from axial spondyloarthritis to the known increased risk of cardiovascular morbidity. Literature on positron emission tomography imaging together with computed tomography (PET/CT) in the context of spondyloarthritis is limited.

Objectives: The aim was to grade the inflammatory signals in the sacroiliac joints and aorta in the PET/CT imaging before and after antirheumatic treatment of clinically active axial spondyloarthritis with either sulfasalazine (SSZ), the firstline antirheumatic drug in Finland for axial spondyloarthritis, or adalimumab (ADA).

Methods: Fourteen patients aged 18-75 years with axial spondyloarthritis and radiologic sacroiliitis as detected either by MRI or X-ray and Bath Ankylosing Spondylitis Disease Activity Index (BASDAI) and visual analogue scale (VAS) $\geq 4$ have been recruited to the pilot study. DMARD-naïve patients started SSZ for 12 weeks. Those who failed to reach remission (BASDAI and VAS $\geq 4$ ) with $S S Z$ or were known to be resistant to conventional DMARDs before inclusion, adalimumab was started for 16 weeks. The patients were scanned with $18 \mathrm{~F}$-fluorodeoxyglucose (FDG) PET/CT after inclusion and after treatment with SSZ and/or ADA. Target-to-background ratio (TBR) was assessed from the aortic root to abdominal aorta as well as for the sacroiliac joints. For statistical comparisons before and after antirheumatic treatment, paired t-test was used. Mean $\pm S D$ are shown.

Results: Our pilot study comprised of nine patients with ADA and five with SSZ. In the SSZ patients (three men, age $36.0 \pm 13.4$ years), mean BASDAI was 4.7 \pm 1.6 before and $3.4 \pm 1.8$ after the treatment $(p=0.091)$. In the ADA patients (six men, age $35.1 \pm 9.8$ years), BASDAl decreased from $4.9 \pm 1.2$ to $2.3 \pm 1.8$ $(p=0.004)$. Among the SSZ patients, maximal TBR in sacroiliac joints declined from $2.35 \pm 0.55$ to $1.51 \pm 0.22(-35.8 \%, p=0.03)$ (figure 1$)$. Aortic maximal TBR declined from $2.44 \pm 0.46$ to $2.07 \pm 0.43(-15.0 \%, p=0.23)$. In the ADA patients, maximal TBR in sacroiliac joints was $1.89 \pm 0.37$ before and $1.90 \pm 0.51$ after treatment $(+0.3 \%, p=0.97)$, and in aorta $2.15 \pm 0.54$ before and $2.37 \pm 0.53$ after treatment $(+10.4 \%, p=0.19)$.

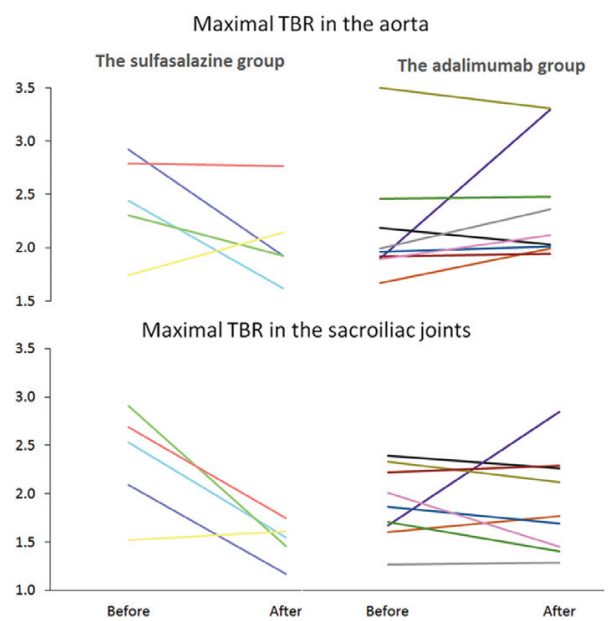

Abstract AB0838 - Figure 1. Maximal TBR in PET/CT in sacroiliac joints and aorta are shown before and after treatment with either sulfasalazine (five patients) or adalimumab (nine patients). Each colour depicts one unique patient.

Conclusions: As detected by reduced 18F-FDG uptake, SSZ reduced inflammation in sacroiliac joints. There was also clear trend towards reduction of inflammation in aorta. ADA and SSZ both reduced clinical symptoms, but only ADA reached statistical significance. PET/CT could not show any reduction in FDG uptake in the ADA group. This may be due to the fact that ADA was used as the second line treatment, when the inflammation had already been reduced to the limit detectable by the method.

Acknowledgements: This study was funded by Abbvie inc.

Disclosure of Interest: J.-P. Kaijasilta: None declared, A. Kerola: None declared, R. Tuompo: None declared, M. Kauppi: None declared, H. Relas: None declared, A. Loimaala: None declared, H. Koivu: None declared, J. Schildt: None declared, T. Kerola: None declared, K. Eklund: None declared, T. Nieminen Grant/research support from: Abbvie Inc

DOI: 10.1136/annrheumdis-2018-eular.4627

\section{AB0839 RADIOLOGIC PARAMETERS OF ANKYLOSING SPONDYLITIS PATIENTS TREATED WITH ANTI-TNF-A VERSUS NON-STEROIDAL ANTI-INFLAMMATORY DRUGS AND SULFASALAZINE}

S.M. Son ${ }^{1}$, J.S. Lee ${ }^{2}$, T.S. Goh², W. Park2 . 'Orthopaedics, Pusan National University Yangsan Hospital, Yangsan; ${ }^{2}$ Orthopaedics, Pusan National University Hospital, Busan, Korea, Republic of Ireland

Background: The introduction of anti-tumour necrosis factor- $\alpha$ (anti-TNF- $\alpha$ ) has significantly altered the treatment landscape of inflammatory arthritis. It has proven to be an excellent treatment option for reducing Ankylosing spondylitis(AS) symptoms. The impact of anti-TNF- $\alpha$ on the radiographic progression of AS has been difficult to characterise, in part because of the relatively slow rate of radiographic change in AS and the hurdles it imposes on longer-term placebo-controlled trials. Despite symptomatic improvement, conclusions concerning effect of anti-TNF- $\alpha$ treatment on radiographic progression in patients with AS remain inconsistent. Furthermore, while many studies have reported the impact of antiTNF- $\alpha$ on radiographic progression, limited data are available on the relationship between treatment agents and sagittal balance in AS.

Objectives: Limited data are available on the relationship between treatment agents and sagittal balance in AS. We investigated radiological features related to treatment agents and compared sagittal balance between patients treated with anti-tumour necrosis factor- $\alpha$ (anti-TNF- $\alpha$ ) or non-steroidal anti-inflammatory drugs (NSAIDs) and sulfasalazine.

Methods: We prospectively enrolled 133 consecutive AS patients. Patients were eligible for the trial if they were under medical treatment with the same treatment agents for at least 1 year. All patients were treated initially with NSAIDs and 